\title{
La Constitución del Ecuador del año 1998 y el año 2008, en la formulación de la política exterior
}

\section{Incidence of the Constitutions of Ecuador of 1998 and 2008, in the Formulation of Foreign Policy}

Henry Nelson Larrea Torres. ${ }^{1}$, Nikolay Alberto Pangol Torres. ${ }^{2}$ \& Lenin Gerardo Larrea Torres. $^{3}$

\section{DOI: https://doi.org/10.33262/visionariodigital.v3i3.670}

\begin{abstract}
The present investigation tries to recognize the incidence of the constitutional norms in the formulation of foreign policy, for that, we start from a central thesis that is defended through its lines, through constant dialogue between authors and students of foreign policy. Initially, a brief analysis is made of the origin and development of foreign policy -transversal elements throughout the documentthrough the vision of academics such as Hill, Jervis, Hudson, Norris, among others, who have deepened the theoretical part of the subject. Subsequently, the development and perspectives of Latin American foreign policy are pointed out through a brief but profound analysis, observing the main schools and theories of thought in force in the region that have been implemented with different impact. Thirdly, we conducted an in-depth study of Ecuador's foreign policy, taking as a framework the Political Constitution of 1998, observing its development and characteristics in the so-called neo-liberal period, whose model was to appear to
\end{abstract}

\footnotetext{
${ }^{1}$ Ministerio de Relaciones Exteriores y Movilidad Humana, hlarreat@cancilleria.gob.ec

2 Investigador Independiente, nikodelatroba1@gmail.com

${ }^{3}$ IESS Hospital General Ambato, llarreat@iess.gob.ec
} 
the authors to be deepened by said normative body. Later we analyzed the Ecuadorian foreign policy from the Constitution of 2008, the date on which the approval and publication of the current Constitution of Ecuador takes place. In this perspective, within this document we address the change of vision set forth in both supreme standards, their background, development and perspectives, all in order to determine whether these standards have affected the formulation of Ecuador's foreign policy. Finally, some conclusions are proposed that, although they do not intend to exhaust the research topic, however, they try to outline important ideas within the reflection to be taken into account within the approach of the Ecuadorian foreign policy.

Keywords: Constitution of Ecuador, Ecuadorian foreign policy, Latin America.

\section{Resumen}

La presente investigación pretende reconocer la incidencia de las normas constitucionales en la formulación de política exterior, para aquello, partimos de una tesis central la misma que es defendida a través de sus líneas, mediante el diálogo constante entre autores y estudiosos de la política exterior. Al inicio se realiza un breve análisis sobre el origen y desarrollo de la política exterior elementos transversales dentro de todo el documento- mediante la visión de académicos como Hill, Jervis, Hudson, Norris entre otros, quienes han profundizado la parte teórica de la materia. Posteriormente, se señala el desarrollo y perspectivas de la política exterior latinoamericana a través de un somero pero profundo análisis, observando las principales escuelas y teorías de pensamiento vigentes en la región y que han sido implementadas con diverso impacto. En tercer lugar, efectuamos un estudio profundo de la política exterior del Ecuador tomando como marco la Constitución Política de 1998, observando su desarrollo y características en el periodo denominado neo-liberal cuyo modelo fue a parecer de los autores profundizado por mencionado cuerpo normativo. Posteriormente analizamos la política exterior ecuatoriana a partir de la Constitución del 2008, fecha en la que se produce la aprobación y publicación de la actual Constitución del Ecuador. En tal perspectiva, dentro del presente documento abordamos el 
cambio de visión planteado en ambas normas supremas, sus antecedentes, desarrollo y perspectivas, todo aquello a fin de determinar si mencionadas normas han incidido en la formulación de la política exterior del Ecuador. Finalmente, se proponen algunas conclusiones que, si bien no pretenden agotar el tema de investigación, sin embargo, intentan esbozar ideas importantes dentro de la reflexión a tomar en cuenta dentro del abordaje de la política exterior ecuatoriana.

Palabras Claves: Constitución del Ecuador, política exterior ecuatoriana, Latinoamérica.

\section{Introducción}

\section{Política Exterior a Nivel Global - Diversas Miradas}

Si partimos de la definición realizada por Christopher Hill sobre política exterior, encontramos que la misma es:

"La suma de relaciones oficiales externas conducidas por un actor independiente (usualmente un Estado) en relaciones Internacionales.” (Hill, 2003, pág. 3).

Sin embargo, dicho concepto no aborda la totalidad de los elementos que encierran la política exterior como tal, siendo necesario también reconocer la existencia de otros actores dentro del contexto internacional.

En contraposición a mencionada mirada, tenemos la visión de Morgenthau quien señala que:

"A political realist, saw international politics as determined by the interest of independent states defined in terms of power." (Adam, 1992, pág. 9),

Según mencionada escuela realista de relaciones internacionales, la política internacional y las relaciones internacionales se reducen -entre otras cosas- al "poder e intereses" de los estados dentro de un sistema internacional naturalmente anárquico.

En efecto, las relaciones internacionales y por ende la política exterior, no puede existir sin sus principales actores (Estados), pero cabe aclarar que estos elementos no son los únicos a decir de Hill:

\footnotetext{
${ }^{4}$ Entiendo que el autor mira las políticas internacionales determinadas por los intereses de Estados independientes definidos en términos de poder.
} 
"The estate is one of a variety of different international actors, whose position relative to each other and to structures needs to be traced." (Hill, 2003, pág. 16).

Por tanto, advertimos la existencia de variedad de actores, niveles y ambientes de diversa índole, como son, las comunidades o actores domésticos; líderes locales y otros cuyo rol de influencia varía según su peso y presencia en la formulación de la política exterior.

En tal sentido, la política exterior entendida desde la visión neorrealista, divisa al sistema internacional articulado en una suerte de Organismos Internacionales pero sostenido por intereses en términos de poder.

Por ello, la mirada de interdependencia compleja de Keohane y Nye señala:

"Los regímenes internacionales proporcionan el entramado politico dentro del que ocurren los procesos económicos internacionales". (KEOHANE, 1998).

Nos permitimos intentar dicha idea, a través de la siguiente cita pues así, por ejemplo:

"vemos a la OMC proporcionando el entramado político donde ocurre el comercio internacional, bajo un modelo de régimen con orientación de mercado" (Oddone \& Granato, 2005)

Todos esos elementos, influyen de diversa manera en la formulación de política exterior. Para varios autores, sin embargo, las percepciones desempeñan un rol muy significativo, es decir, su importancia radicaría -en política exterior-, en que aquellas pueden determinar e influir en las diversas etapas de su construcción, otorgándoles peso y legitimidad a los tomadores de decisiones, otorgando peso así también, a la lógica sistémica en la que se crean.

En señalada línea de pensamiento, algunos autores sostienen que:

"logic alone cannot tell us that a similar claim about the decision-making process is invalid: the way people perceive others a make decision only marginally influences outcomes." 6 (Jervis, 1976, pág. 14)

En tal contexto, las diversas visiones de política exterior visualizan a dicha ciencia como un todo interrelacionado, en el que, participan actores de diversa índole entre los que se

\footnotetext{
${ }^{5}$ Entiendo que el autor ve al Estado como uno de los diferentes actores de las relaciones internacionales formando parte de la estructura internacional.

${ }^{6}$ Entiendo que, el autor mira a las percepciones como parte importante en la toma de decisiones y los resultados que de estas derivan dado que la simple lógica no puede explicar la política exterior en su totalidad.
} 
encuentran los Estados ${ }^{7}$, los mismos que responden a un cúmulo de factores como son la economía, la cultura, las relaciones de poder (local-global) así como los elementos mediáticos que afectan indicadas relaciones de poder dentro de dichos territorios y en sus relaciones con otros estados.

Según dicha visión:

"Too often public discussion oscillates between fatalism about the impossibility of affecting international affairs, and the personalization of policy through the high expectations held of individual leaders." (Hill, 2003, pág. 19)

En síntesis, la política exterior a nivel global ha sido vista y aplicada desde varias teorías y escuelas de acuerdo al enfoque teórico al que obedece su estudio, partiendo desde visiones clásicas como el realismo, el constructivista, el neo-marxista o teoría crítica, la interdependencia compleja, así como revisiones al realismo clásico o visión neo-realista. Sin embargo, y para para encaminar teóricamente el presente documento, he profundizado más mi mirada en una visión que plantea la formulación de la política exterior desde una visión mayormente matizada con elementos constructivistas.

\section{Política Exterior en América Latina}

\section{Breve análisis}

La política exterior latinoamericana, conserva casi intacta su raíz y visión colonial, posterior a la independencia. Por ello, según lo sostienen varios autores, las luchas por la independencia más que una disputa en contra de los centros:

“metrópolis lejanas, se convertía en verdaderas guerras civiles en contra de los poderes locales cercanos" debido a que "En 1808-1809, el proyecto de independizarse de España estaba lejos de contar con la aceptación de un número significativo de americanos" (Démelas, 2009, pág. 28)

\footnotetext{
${ }^{7}$ Sin lugar a duda.

${ }^{8}$ En este sentido el autor sostiene la importancia de los liderazgos personales en el debate de formulación de política exterior, al señalar que: "el debate público oscila entre el fatalismo acerca de la imposibilidad de afectar a los asuntos internacionales, y la personalización de la política a través de las altas expectativas de los líderes individuales"
} 
Dicha influencia -colonial- se observaría a lo largo y ancho del continente americano cuyas ex-colonias, sostenían costumbres e incluso instituciones de sus anteriores colonizadores, así vemos que:

"A pesar de haber proclamado su independencia, las provincias unidas del Río de la Plata seguían distinguiendo villas y ciudades estos dos estamentos de la nobleza ciudadana del antiguo régimen" (Démelas, 2009, pág. 35)

Luego de la secesión de "La Gran Colombia"9 muchos países de la región interandina entre ellos Ecuador- sostienen en sus legislaciones una estructura de la tradición postcolonial, provocando que el nuevo estado se convierta en lo que algunos autores consideran como un "estado colchón"10

Durante el siglo 20, los países latinoamericanos se introducen a la división internacional del trabajo. La política exterior en tal sentido, profundizó modelos primario exportadores los mismos que se han sostenido en la mayoría de naciones de la región hasta nuestros días, provocando diferencias extremas con los países llamados "de primer mundo" lo que agudizo la división de estados en "centrales -periféricos".

Por otra parte, a partir de la década de los 60:

"Dentro del contexto mundial, a nivel global se produce una reestructuración del sistema económico que hasta aquel momento se enfocaba en políticas Keynesianas, (...) que buscaba legitimar la intervención redistribuidora del Estado" (ECHEVERRÍA, 2006, págs. 16,17)

Ya en éste último siglo, la política exterior en Latinoamérica, se encontró muy vinculada a la agenda estratégica planteada por los Estados Unidos para la región, así observamos, como grandes intereses económicos de los denominados centros se entrecruzan dentro de los conflictos entre países americanos, primero al aliarse en la lucha en contra del comunismo, durante todo el proceso de guerra fría y posteriormente al reflejar -a partir de los años 70- los intereses de Organismos Internacionales, principalmente el FMI ${ }^{11}$,

\footnotetext{
9 “En medio del conflicto bélico por la Independencia se constituyó la República de Colombia, formada por Venezuela, Nueva Granada y Quito, que se incorporó posteriormente.” (Ayala, 2008, pág. 107)

${ }^{10}$ Según varios autores, aquel Estado que por encontrarse en medio de dos Estados con mayor fuerza o poder, buscan acoplarse a la política de dichos estados en lucha por su sobrevivencia.

${ }^{11}$ Fondo Monetario Internacional
} 
$\mathrm{BM}^{12}$ así como la $\mathrm{OMC}^{13}$, cuyo rol es de vital importancia para entender el enfoque con el cual ha sido alimentada la formulación de política exterior a nivel de la región, durante las últimas décadas.

Mencionados organismos internacionales, han intervenido en la mayoría de países de la región implantando una visión hegemónica, cuya principal experiencia la vivió Chile a partir de la dictadura militar, en tal sentido:

"Merece la pena recordar que la instauración del primer experimento de formación de un estado neoliberal se produjo en Chile el 11 de septiembre de 1973” (Harvey, 2010, pág. 15)

Según dicho autor, las diversas versiones -de estado neoliberal- han sido implementados gradualmente, y con diversa intensidad en la política exterior de los países de América Latina, política que incluso sigue latente en varios Estados de la región como es el caso de Colombia ${ }^{14}$, cuyo apéndice central obedecería en gran medida a la ya mencionada agenda estratégica norteamericana, la misma cuyo principal interés se encuentra centrado en temas de "seguridad, combate al terrorismo y democracia".

Desde la misma óptica de pensamiento, siguiendo detenidamente a Walter Martínez, la política militar de EEUU, está enfocada en una estrategia oficial de largo plazo. Según señalado autor, dicha política explicaría el avance y desarrollo de bases militares norteamericanas en la región, las mismas que han crecido significativamente, por lo tanto, dice:

"Sin contar el caso de Puerto Rico, son por lo menos 47 bases militares extranjeras en funcionamiento, o en construcción vinculadas por vía aérea y marítima con la IV Flota naval reactivada desde 2008." (Martínez, 8 de mayo de 2012.).

Es necesario señalar, que según dicho autor -al igual que otros-, el incremento de la presencia militar norteamericana en la región los últimos años, obedece a factores

\footnotetext{
${ }^{12}$ Banco Mundial

${ }^{13}$ Organización Mundial de Comercio.

${ }^{14}$ La Implementación de 8 a 16 bases militares norteamericanas en dicho país, dan cuenta de la vinculación de la política exterior con la agenda de seguridad planteada por Norteamérica.
} 
geopolíticos y geoestratégicos debido a la gran cantidad de reservas de minerales y a la ubicación privilegiada de América Latina en el contexto mundial.

Por ende, al parecer creería necesario proponer dentro de los procesos de integración una agenda que fortalezca los mecanismos de conservación y aprovechamiento de nuestros recursos, cambiando la matriz productiva de nuestros países (conforme el inmenso esfuerzo que vienen desarrollando algunos países entre ellos Ecuador), respetando al ser humano y la naturaleza.

Para todo ello es necesario tomar en cuenta que:

"Toda presencia militar estadounidense en nuestro territorio y mares es altamente peligroso y debe ser rechazada porque resta fuerza a la autodeterminación; y ante la eventualidad de una gran guerra, aquel país latinoamericano y caribeño con una base militar de los EE. UU corre el riesgo de ser atacado." (Dierckxsens, 2011)

\section{Política Exterior ecuatoriana durante la vigencia de la Constitución de 1998}

\section{Antecedentes:}

La Constitución de la República del Ecuador de 1998, nace en un contexto de crisis generalizada a nivel de Latinoamérica, debido -entre otras cosas- a las medidas aplicadas en 1994 derivadas del llamado Consenso de Washington ${ }^{15}$ a través del cual los Organismos de Bretton Woods ${ }^{16}$ fortalecen su importancia a nivel mundial al implementar las denominadas cartas de intención ${ }^{17}$, o instrumentos comerciales, mismos que sumieron a muchos Estados en crisis económicas que desatarían en problemas sociales considerables, años más tarde y cuyas secuelas aún son palpables.

En el caso específico ecuatoriano, encontramos un sinnúmero de reformas que se venían plantando ya desde inicios de la década de los noventa, así observamos, que uno de los

\footnotetext{
15 Se denominan así, las decisiones tomadas en el Estado de Washington en Estados Unidos, en donde se plantearon las denominadas "recetas financieras" para salir de la crisis generalizada en la que se sumergen varias regiones del mundo, mencionadas recetas fueron enviadas especialmente a Latinoamérica.

16 Banco Mundial, Fondo Monetario Internacional y la Organización Mundial del Comercio anterior GATT.

${ }^{17}$ Mecanismos de aplicación económica según los cuales los Estados se comprometían a realizar varias reformas económicas a fin de recibir recursos del FMI.
} 
elementos generalizados, no solo en Ecuador, sino en toda la región, fue la disminución del tamaño del Estado mediante mecanismos denominados privatizaciones, la liberalización de mercados y la flexibilización laboral, así como la financiera.

No debemos dejar a un lado, que en dicho periodo observamos que:

"Los procesos de reforma institucional neoliberal, dan un duro golpe a la planificación estratégica nacional, pues se asiste, a mediados de los años 90 a la transición del Consejo Nacional de Planificación, en ODEPLAN, oficina dependiente de la Presidencia de la República.” (ECHEVERRÍA, 2006, pág. 28).

Dentro de aquel escenario, y con un Estado totalmente debilitado por las reformas planteadas, nacen cronológicamente leyes que aceleran el proceso neoliberal en Ecuador, entre ellas: la Ley de Modernización del Estado y Privatizaciones del Sector Público, Ley Reformatoria del Sistema Financiero y con posterioridad a inicios del siglo XXI; la Ley de Transparencia y Estabilidad Fiscal del año 2002 en cuyo texto se creó el FEIREP ${ }^{18}$ al mismo tiempo que se estableció un máximo de inversión pública de un $4.5 \%$ del PIB para las instituciones públicas.

Podemos observar también (a manera de ejemplo), como en Ecuador durante la época denominada neoliberal,

"el ciclo de políticas (entre ellas la política exterior) obedeció a un enfoque de liberalización del mercado por tanto respondía -en algunos casos- a normas impuestas por organismos transnacionales como el FMI y el Banco Mundial” (Larrea, 2012, pág. 2).

Por tanto, la política exterior del Ecuador recogió de esta matriz "neoliberal” su principal inspiración y eje central cuyo horizonte planteaba la libre circulación de mercancía, así como de capitales y en contrapartida, plantea trabas, así como mayores requisitos a la circulación de seres humanos.

Por ello, si damos una mirada retrospectiva a la formulación de política exterior ecuatoriana, debemos considerar y tomar en cuenta, visiones planteadas desde varios académicos quienes sostienen (refiriéndose a la década pasada) que durante la década de los noventa:

\footnotetext{
${ }^{18}$ Fondo de Estabilización Inversión Social y Productiva y Reducción del Endeudamiento Público
} 
"La política exterior ecuatoriana en los últimos 10 años no ha existido, si queremos por ello entender una estrategia consistente de participación del Estado en las relaciones internacionales" (Jaramillo, 2008, pág. 1)

No sorprende entonces, ver como las relaciones comerciales a las que tendía la "política exterior" de los años noventa y el primer lustro de la década siguiente tuviera como eje central el fortalecimiento de las relaciones con los países del norte, pero principalmente con EEUU, así se observaba en aquel entonces que:

“La economía política del Ecuador, donde el $48 \%$ de sus exportaciones van hacia Estados Unidos, ha marcado el camino también de la política exterior" (Jaramillo, 2008, pág. 1)

De igual manera al resto de países de la región, advertimos como Ecuador ve marcada su política exterior en las relaciones sur-norte pues:

"La búsqueda de cooperación estadounidense generaba a su vez procesos de alineamiento político/diplomático con la agenda estadounidense en materia de drogas, seguridad y ahora terrorismo y la versión liberal de democracia." (Jaramillo, 2008, pág. 2)

En virtud a que:

"Los temas de la agenda internacional de un país pequeño y débil se construyen generalmente fuera de su entorno." (Bonilla, 2008, pág. 3)

Vemos pues, que durante mencionado periodo y hasta el 2006, el proceso de construcción de la política exterior ecuatoriana, fue marcado por la visión de realismo clásico, así como el sentimiento y/o percepción de Estado débil, “Estado colchón” y pequeño.

Por tanto:

"La clave del proceso de implementación del modelo neoliberal en Ecuador, fue la aprobación de la Constitución de 1998, que premiaría entre sus artículos el saqueo de recursos y el debilitamiento del Estado por medio de las privatizaciones a través de la "economía social de mercado" reconocida en su artículo 244: así como la posibilidad de créditos del Estado a bancos privados que se encuentren en "problemas" conforme la disposición transitoria $N^{\circ} 42$ de la Norma Suprema. ( Asamblea Constitucional, 1998). “Esto impidió la intervención del Estado como 
ente regulador económico y prestador de servicios, provocando un sistema político clientelista. Todo aquello generó una política exterior alejada de las bases, forzando a la ciudadanía a la movilización en una constante lucha social callejera (participación informal) en virtud a conseguir mejores mecanismos en la toma de decisiones y representación.” (LARREA TORRES, 2012)

En síntesis, en la época estudiada la política exterior ecuatoriana, respondía -como se ha señalado- en gran medida a intereses extranjeros y no al necesario beneficio nacional.

\section{Política Exterior en Ecuador a partir de la visión planteada en la Constitución del 2008}

A diferencia del modelo neoliberal definido en el artículo 244 de la Constitución Política del Ecuador de $1998^{19}$, en la nueva Carta Magna ecuatoriana publicada el 20 de octubre de $2008^{20}$, se genera un profundo cambio de ruta en lo que a enfoque de formulación de políticas públicas en general y política exterior en especial se refiere.

Entre las acciones específicas que dan cuenta de aquello, se encuentran entre otras, que el cambio de eje central de la política exterior ecuatoriana, giró en torno al fortalecimiento de las relaciones sur-sur a diferencia de las anteriores tendencias existentes sur-norte.

Así también, en el artículo 5 de la Carta Magna del 2008, se menciona, por ejemplo:

"se prohíbe el establecimiento de bases militares extranjeras en todo el territorio ecuatoriano"

La visión de soberanía vista desde esa perspectiva, se ve fortalecida.

Por otra parte, el artículo 416 de la Constitución establece -uno tras otro- los principios de la nueva visión en las relaciones internacionales del Ecuador, entre lo más destacado tenemos el tema de la “ciudadanía universal” (Asamblea Constituyente, 2008), así como, el fortalecimiento de la integración regional.

19 En mencionado artículo la Constitución sostiene "Art. 244.- Dentro del sistema de economía social de mercado al Estado le corresponderá: 1. Garantizar el desarrollo de las actividades económicas, mediante un orden jurídico e instituciones que las promuevan, fomenten y generen confianza." ( Asamblea Constitucional, 1998)

${ }^{20}$ Constitución cuyo eje central se lo denomina "Sumak Kausay" o Buen Vivir, misma que a decir de varios autores abandona la visión mirada "mercado-céntrica" implementado 'por la Constitución de 1998. 
Haciendo eco de señaladas normas constitucionales, el Plan Nacional de Desarrollo, establece en su objetivo 5, un sinnúmero de proyectos, programas y metas a fin de profundizar las políticas, con el propósito de cumplir metódicamente los objetivos estratégicos de la planificación nacional, para ello señala que el Ecuador deberá actuar:

"privilegiando la inserción soberana del Ecuador con el resto del mundo priorizando la integración latinoamericana ${ }^{21}$ " (SEMPLADES, 2012)

En la misma línea hace algún tiempo atrás, se señalaba que:

"Ecuador ha sido precisamente el país que más entusiasmo ha puesto en los últimos 10 meses en la conformación de la Unión de Naciones del Sur (UNASUR), entidad sudamericana que sucedería a la Comunidad Sudamericana de Naciones (CSN), que se había conformado a raíz de la Declaración de Cuzco de diciembre del 2004" (Jaramillo, 2008, pág. 3)

Dentro de mencionada visión, y con afán de profundizar las relaciones con países de la región, Ecuador ingresa el año 2009 al $\mathrm{ALBA}^{22}$, organismo regional, que para varios autores es una alternativa a la integración planteada años atrás por Clinton a través del ALCA cuya matriz era estrictamente comercial.

Por tal motivo, podemos encontrar que para varios autores:

"La propuesta del ALBA radica sobre cuatro elementos básicos: la complementariedad, la cooperación, la solidaridad y el respeto a la soberanía y la autodeterminación de los pueblos” (Vazquez S, Saltos G, 2012, pág. 77). En virtud a que: "El ALBA parte de la integración, en primera instancia, desde lo político y desde lo social. "23

Al respecto de las relaciones internacionales, la nueva visión de la política exterior ecuatoriana pretende profundizar las relaciones con países no solo de América, sino además de otros continentes principalmente con países del Asia, como la China, Corea, y otros como Irán y Turquía, destinos cuyas relaciones internacionales se han fortalecido los últimos años.

\footnotetext{
${ }^{21}$ Plan Nacional de Desarrollo y Buen Vivir 2009-2013.

${ }^{22}$ Alianza Bolivariana para América

${ }^{23}$ IBIDEM. (Vazquez S, Saltos G, 2012, pág. 78)
} 
Sin embargo, a todo lo dicho dentro de los destinos tradicionales de relacionamiento vemos que:

"La nueva visión de la política exterior ecuatoriana tiene también una nueva preferencia: acelerar las negociaciones comerciales con la Unión Europea y hacer de la UE un socio estratégico alternativo, frente a Estados Unidos. Es curioso que a pesar de la similitud de los acuerdos comerciales que se negocian actualmente con el fallido TLC con Estados Unidos, el gobierno ecuatoriano haya optado por acelerar el paso en las negociaciones andinas conjuntas con la Unión Europea” (Jaramillo, 2008, pág. 4)

Por todo lo señalado es importante recordar que:

"La constatación de nuestra realidad actual puede provocar pesimismo o desencanto de nuestras posibilidades hacia el futuro. Pero no cabe duda de que hay entre nosotros energías positivas. El acuerdo de paz con el Perú y la integración andina y sudamericana abren grandes posibilidades. Ciertamente, la compleja situación que vivimos es al mismo tiempo una motivación para que la superemos." (Ayala Mora, 2008, págs. 43,44)

En síntesis, durante el presente trabajo hemos observado, el cómo la política exterior ecuatoriana ha ido variando significativamente su enfoque, el mismo que ha sido influido en forma directa por las normas constitucionales.

Si bien es cierto, el tema en cuestión no se lo puede agotar en un estudio de esta naturaleza, el objetivo principal del mismo es sin embargo dar unas breves orientaciones y perspectivas de la incidencia de las normas supremas en la formulación de la política exterior ecuatoriana.

\section{CONCLUSIONES:}

- La política exterior ecuatoriana durante el periodo de ingreso a la democracia, hasta la vigencia de la Constitución del año 1998, obedeció a un enfoque neoliberal cuyo centro reflejaba una visión mercado-céntrica, la misma que debilitó profundamente las estructuras estatales.

- La política exterior ecuatoriana durante el periodo de vigencia de la Carta Magna de 1998, por su visión y enfoque, no reflejaba los intereses nacionales ni la 
democratización en la toma de decisiones, al contrario, señaladas normas favorecían a intereses de organismos internacionales, así como a transnacionales representantes de estados céntricos, los mismos que buscaban a través del neoliberalismo encontrar nuevos mercados para sus productos.

- La visión de estado colchón, empieza a debilitarse con la nueva Constitución 2008, incluso un poco antes al haber superado el conflicto limítrofe entre Ecuador y Perú.

- Con la Carta Magna del año 2008, la política exterior ecuatoriana cambia de rumbo al dar preponderancia al fortalecimiento de las relaciones sur-sur, así como, al premiar la integración latinoamericana, lo que se ve reflejado con la diversidad de organismos regionales que se han creado en los últimos años, así como, en la diversidad de acuerdos, tratados y convenios firmados con nuestros países vecinos y con otros cuyas relaciones no han sido tradicionales.

\section{Bibliografía}

Asamblea Constitucional. (1998). Constitución Política del Ecuador 1998. (R. O. Ecuador, Ed.) Quito, Ecuador: Registro Oficial Ecuador.

Adam, W. (1992). The Evolution of the International Society London and New York Routledge. London and New York Routledge, EEUU.

Asamblea Constituyente. (2008). Constitución del Ecuador. Quito, Pichincha, Ecuador: Registro Oficial Ecuador.

Ayala Mora, E. (1 de Agosto de 2008). Resumen de Historia del Ecuador. (C. E. Nacional, Ed.) Recuperado el 19 de enero de 2013, de http://repositorio.uasb.edu.ec/bitstream/10644/836/1/AYALAE-CON0001RESUMEN.pdf

Ayala, E. (2008). “La Nación Ecuatoriana en la Historia”. En B. A.-M.-I. Internacional, Ecuador miradas binacionales (págs. 105-118). Bogotá: S. Ramírez (Ed.). 
Bonilla, A. (2008). 25 años de vulnerabilidad (Vol. Ensayo no publicado). Quito: FLACSO-Sede Ecuador.

DE LA TORRE, C. (2003). Los usos políticos de las categorías pueblo y democracia. En E. S. Pachano, Antología. Ciudadanía e identidad (págs. 231-248). Quito: FLACSO.

Démelas, M.-D. (2009). “Separar y unir: algunas preguntas sobre la formación de las nuevas naciones andinas y sus relaciones a principios de la independencia” en Ecuador. En C. B. Zepeda, Relaciones Internacionales a la luz del Bicentenario (págs. 27-43). Quito: FLACSO.

Dierckxsens, W. (2011). Siglo XXI, Crisis de una Civilización. Quito: IAEN.

Echeverría, J. (2006). Crisis de gobernabilidad y legitimidad en los años 80. En J.

Echeverría, En El desafío constitucional, (págs. págs. 9-35, 123-145). Quito: Abya-Yala.

ECHEVERRÍA, J. (2006). El Desafio Constitucional (Primera ed.). (ILDIS-FES, Ed.) Quito, Pichincha, Ecuador: ABYA YALA.

Espinosa, C. (1999). “La negociación como terapia,” El Proceso de Paz Ecuador-Perú. Quito: FLACSO-Fundación Kellog.

Harvey, D. (2010). Breve historia del neoliberalismo. Versión electrónica.

Hill, C. (2003). The changing politics of foreign policy. New York: Palgrave MacMillan.

Jaramillo, G. (1 de junio de 2008). "Escenarios Divergentes de la Inserción Ecuatoriana en el Mundo". (C. d. CIDOB, Ed.) DCIDOB.

Jervis, R. (1976). Perception and misperception in international politics. New Jersey: Princeton University Press. 
Keohene, R. Y. (1998). Poder e Interdependencia- La política mundial en transición. Buenos Aires: Grupo Editor Latinoamericano.

Laclau, E. (2011.). Populismo: ambigüedades y paradojas. En La razón populista, Buenos Aires: Fondo de Cultura.

Larrea Torres, H. (01 de enero de 2012). La Constitución del Estado Ecuatoriano y la participación ciudadana en el periodo 1998-2010. Ensayo Final de la Materia El Estado Ecuatoriano. Quito, Pichincha, Ecuador: Inédito.

Larrea, H. (20 de agosto de 2012). Reseña N² Cátedra de Historia Política e Institucional del Ecuador. Quito, Pichincha, Ecuador-Inédito.

Martínez, W. (8 de mayo de 2012.). Nuevas estrategias militares y de intervención del Imperio en Nuestra América. En V. S. en (Ed.), Ponencia presentada por el Brig. Gral.(R) Walter Martínez en el V, (págs. 1-52). México, D.F.

Oddone, M., \& Granato, L. (18 de noviembre de 2005). Un Régimen para el Comercio Internacional, Recuperado el 06 de diciembre de 2012, de http://www.eumed.net/ce/2005/nolg-omc.htm

SEMPLADES. (2012). PLAN NACIONAL DEL BUEN VIVIR. 5 años de Revolución Ciudadana, 28-29.

Sherif, R. (2012). Parte 1 Mujer al Frente., (pág. 11). Quito.

(2012). En L. Vázquez S, Saltos G, Ecuador su realidad (pág. 18). Quito: Fundación de Investigación y Promoción Social "José Peralta".

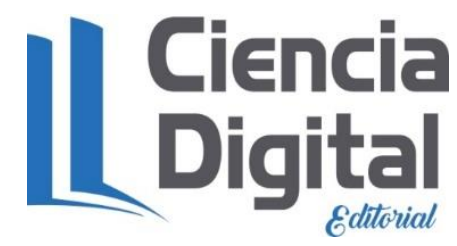




\section{PARA CITAR EL ARTÍCULO INDEXADO.}

Larrea Torres, H., Pangol Torres, N., \& Larrea Torres, L. (2019). La Constitución del Ecuador del año 1998 y el año 2008, en la formulación de la política exterior. Visionario Digital, 3(3), 206-222.

https://doi.org/10.33262/visionariodigital.v3i3.670

\section{Ciencia
Digital
Édtabal}

El artículo que se publica es de exclusiva responsabilidad de los autores y no necesariamente reflejan el pensamiento de la Revista Visionario Digital.

El artículo queda en propiedad de la revista y, por tanto, su publicación parcial y/o total en otro medio tiene que ser autorizado por el director de la Revista Visionario Digital.
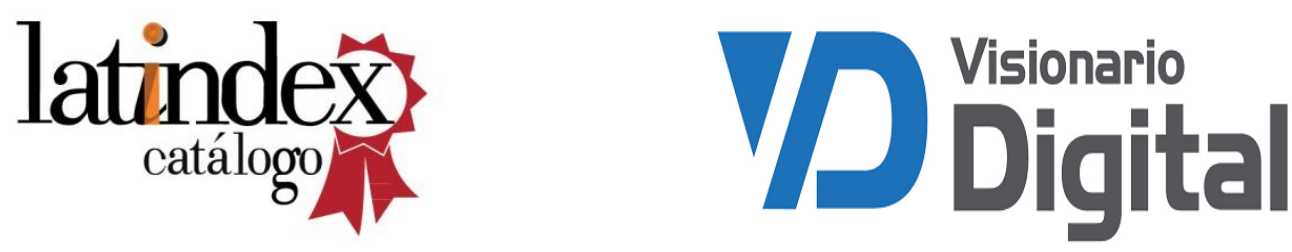